\title{
Determination of Optical Positions for Extragalactic Radio Sources under the Collaboration Between SHAO and NAO
}

\author{
Z. H. Tang, W. J. Jin and S. H. Wang \\ Shanghai Astronomical Observatory, Chinese Academy of Sciences, \\ Shanghai 200030, China \\ G. Pinigin, A. Shulga, N. Maigurova, Yu. Protsyuk
Nikolaev Astronomical Observatory, Nikolaev, 327030, Ukraine
}

\begin{abstract}
The optical positions of 9 compact extragalactic radio sources have been determined by using the $1.56 \mathrm{~m}, 1 \mathrm{~m}$ and $60 / 90 \mathrm{~cm}$ telescopes with CCDs in China and the Axial Meridian Circle at Nikolaev Astronomical Observatory since Jan. 1996 in a cooperative project between the two observatories. The instrumentation, observations and reduction are briefly described, and the preliminary results are presented. The comparison between the optical and radio positions for 9 sources are also given. More optical positions of radio sources are being processed.
\end{abstract}

\section{Introduction}

With the compact extragalactic radio sources (CERS) as the fiducial points, the radio reference frame may be said to be a quasi-inertial reference frame (Ma et al., 1998). Because the sources are very remote, their proper motions (approximately a few $\mu a s / y r$ ) are negligible as compared with observational accuracy. Since January 1998, the International Celestial Reference System (ICRS) has been adopted by the 23rd IAU General Assembly, and is realized by the International Celestial Reference Frame (ICRF), which is combined from various radio catalogs by the International Earth Rotation Service (IERS). The ICRS is realized by the Hipparcos Catalog in optical wavelengths. The Working Group (WG) on the ICRS was set up at 23rd IAU General Assembly. The WG is concerned with all aspects connected with the approved International Celestial Reference System: its use, extension and its promotion to the astronomical community (Mignard, 1998). The main tasks of the WG are maintenance and extension of the ICRS, densification, and linkage to the dynamical system.

A joint project between Shanghai Astronomical Observatory (SHAO) and Nikolaev Astronomical Observatory (NAO) has been carried out since Jan. 1996 to determine the optical positions of the CERS using the $1.56 \mathrm{~m}$ telescope with CCD at SHAO and the positions of secondary reference stars around the CERS determined with the fully automatic Axial Meridian Circle (AMC) at NAO. The preliminary results are presented and the comparison between the optical and radio positions are given in this paper. 


\section{Instrumentation}

The $1.56 \mathrm{~m}$ reflector is a $\mathrm{R}-\mathrm{C}$ type telescope and the AMC is a horizontal telescope. In order to observe the faint stars the CCD cameras were installed on two telescopes. The optical parameters of the two telescopes are listed in Table 1. Sometimes a corrector for reducing the focal length was used on the $1.56 \mathrm{~m}$ telescope, which makes the focal length three times shorter.

Table 1. Optical parameters of the telescopes.

\begin{tabular}{ccc} 
parameter & $1.56 \mathrm{~m}$ telescope & AMC \\
\hline diameter & $1.56 \mathrm{~m}$ & $180 \mathrm{~mm}$ \\
focal length & $15.6 \mathrm{~m}$ & $2480 \mathrm{~mm}$ \\
scale & $13^{\prime \prime} 25 / \mathrm{mm}$ & $83^{\prime \prime} 19 / \mathrm{mm}$ \\
field of view & $4.4 \times 4^{\prime} 4$ & $23^{\prime} \times 26^{\prime}$ \\
CCD & $1024 \times 1024$ & $1040 \times 1160$ \\
\hline
\end{tabular}

Some other principal components of the AMC follow. The prizm unit was made from a cital cylinder with a central hole diameter $40 \mathrm{~mm}$ truncated at 45 degrees and a diameter of $180 \mathrm{~mm}$. The divided glass circle with the reading system has 4 parameters: diameter of $420 \mathrm{~mm}$, division interval of $5^{\prime}$, four reading microscopes with mean error 0.02 and reading time $16 \mathrm{sec}$. The autocollimator with CCD micrometer in the prime vertical has parameters: objective aperture of $180 \mathrm{~mm}$, focal length of $12360 \mathrm{~mm}$, scale reading in the image plane $1^{\prime \prime}=59.92 \mu \mathrm{m}$. The tube is evacuated to eliminate the refraction anomalies in the horizontal light path. The CCD autocollimator micrometer has an error reading an artificial mark of 0.02 .

In addition, because the weather at the Sheshan station of SHAO is not good enough, other telescopes were also used for this purpose. A $1 \mathrm{~m}$ reflector with a $1024 \times 1024 \mathrm{CCD}$ was used at Yunnan Astronomical Observatory (YNAO). The field of view is $6.5 \times 6.5$ and the focal length is about $13 \mathrm{~m}$. A $60 \mathrm{~cm}$ reflector with a $1024 \times 1024$ CCD was used at Xing-Long Station of the Beijing Astronomical Observatory (Wang et al., 1998). The field of view is $16^{\prime} \cdot 6 \times 16^{\prime} 6$ and the focal length about $2.5 \mathrm{~m}$. A $60 / 90 \mathrm{~cm}$ Schmidt telescope with a $2048 \times 2048$ CCD was used at Xing-Long Station of the Beijing Astronomical Observatory. The field of view is $1^{\circ} \times 1^{\circ}$ and the focal length is $1.8 \mathrm{~m}$ (Tang et al., 1998).

\section{Observations and reductions}

The optical counterparts of 86 compact extragalactic radio sources have been observed in China since Jan. 1996 (see Table 2). There are 36 CERS also observed by the AMC (with the mark *).

The field of view of the CCD is so small that almost all distortions may be ignored. In using the $60 / 90 \mathrm{~cm}$ telescope, only the center area of the $1^{\circ} \times 1^{\circ}$ field of view was used. Therefore the relationship between the measured and the standard coordinates may be expressed by orthogonal linear equations (Murray 1983).

There are three methods: centroid, gaussian and optimum filter in the centering task of the IRAF software used to determine the centers of star images. 
Table 2. 86 compact extragalactic radio sources.

\begin{tabular}{lllll}
\hline $0014+813$ & $0036+030$ & $0055+300$ & $0111+021$ & $0116+319$ \\
$0135-247$ & $0153+744$ & $\mathbf{0 2 1 5}+015$ & $0241+622$ & $0309+411^{*}$ \\
$0316+413$ & $0355+508$ & $0405-123$ & $0420-014^{*}$ & $0430+052^{*}$ \\
$0528-250$ & $0538+498^{*}$ & $0552+398^{*}$ & $0607-157$ & $0636+680^{*}$ \\
$0642+449$ & $0707+476^{*}$ & $0716+714$ & $0718+792$ & $0723-008$ \\
$0735+178^{*}$ & $0736+017$ & $0738+313^{*}$ & $0814+425$ & $0818-128^{*}$ \\
$0820+560^{*}$ & $0827+243^{*}$ & $\mathbf{0 8 3 6}+710^{*}$ & $0839+187$ & $0839+200$ \\
$0850+561$ & $0851+202^{*}$ & $\mathbf{0 8 5 9 - 1 4 0}$ & $0917+449^{*}$ & $\mathbf{0 9 5 5}+326$ \\
$1034-293$ & $1038+064$ & $1039+811$ & $1040+123$ & $1044+719$ \\
$1045-188$ & $1127-145$ & $1128+385^{*}$ & $1144+402^{*}$ & $1145-071$ \\
$1148-001^{*}$ & $1150+497$ & $1216+487$ & $1219+285$ & $1226+023$ \\
$1228+126^{*}$ & $1237-101$ & $1257+145^{*}$ & $1302-102^{*}$ & $1354+195^{*}$ \\
$1404+286^{*}$ & $1418+546^{*}$ & $1419+536$ & $1420+326$ & $1442+101$ \\
$1510-089$ & $1538+149^{*}$ & $.1641+399$ & $1652+398^{*}$ & $1658+053$ \\
$1705+456^{*}$ & $1727+502^{*}$ & $1749+096^{*}$ & $1749+701$ & $1803+184$ \\
$1821+107^{*}$ & $1838+052$ & $1845+797$ & $1901+319^{*}$ & $1937-101^{*}$ \\
$2007+777$ & $2128-123$ & $2145+067^{*}$ & $2200+420^{*}$ & $2251+158^{*}$ \\
$2344+092^{*}$ & & & & \\
\hline
\end{tabular}

The results of test comparisons show that the centroid method is a little better than the other two methods. It should be better to choose the appropriate sampling range to compute the centers of star images, which is based on the magnitude. The reduction method of the AMC in right ascension and declination is the general procedure for a meridian circle.

\section{4. $\mathrm{AMC} 1.0$}

In 1996, the observation program of intermediate reference stars in the fields around 250 northern CERS in the declination zone from $+70^{\circ}$ to $-20^{\circ}$ and in the 12-14 magnitude range was started. The positions of the reference stars, selected from the GSC, were provided in the Hipparcos system to improve the optical/radio reference frames linkage (ESA, 1997). AMC 1.0 was completed in August 1999, in which more than 10000 secondary reference stars around 220 CERS were observed.

The comparison between the results of the AMC and the Hipparcos catalogs and other instruments are shown in Figures 1 and 2 (Kovalchuk et al., 1997). The figures show the variation of the mean systematic differences of $(O-C)_{\alpha} \cos \delta$ and $(O-C)_{\delta}$ for each declination zone in steps of 5 degrees. The errors of the mean systematic differences $(O-C)$ ranges from $0 . " 03$ to $0 . \prime 05$, Results for the AMC show that 1) the internal accuracy of mean systematic differences is about $\left.0{ }^{\prime \prime} 01-0,02,2\right)$ accuracy of one observation for HC stars $0 ! 11$ sec $0.65(Z)$ for both coordinates and about 0 " $13-0$ "' 20 for GSC stars, 3 ) the position accuracy of the AMC catalog is 0.05 . Of course, to improve the precision of the AMC more observations will be needed because some stars have only a single observation. The second version of the AMC will be released soon. 


\section{Results and comparison}

The 9 optical positions of CERS using the second reference stars of AMC are listed in Table 3 in which the name and magnitude of CERS, $\alpha$ and $\delta$ at J2000.0 are listed in columns 1 to 4 , and the observing epoch is listed in column 5 . Though the AMC has no proper motions, the epoch of observations of SHAO and that of the AMC is not more than 3 years, so the influence of proper motions is small. The number of reference stars is about 4 to 9 . The comparisons between optical and radio positions published in IERS Technical Note 23 are listed in columns 6 and 7 of Table 3 . The mean absolute difference of opticalradio positions for 9 CERS is 0.25 and 0.40 in right ascension and declination respectively. More optical positions of CERS are being processed.

Table 3. Positions of nine sources.

\begin{tabular}{|c|c|c|c|c|c|c|}
\hline Name & mag & $\alpha$ & $\delta$ & $\Delta \alpha$ & $\Delta \delta$ & $\mathrm{T}$ \\
\hline & & h $\mathbf{m} s$ & $0=11$ & & & \\
\hline $0552+398$ & 18.0 & 055530.797 & +394849.20 & -0.10 & $=+0.03$ & 1996.16 \\
\hline $0735+178$ & 16.2 & 073807.358 & +174219.29 & -0.50 & $=+0.29$ & 1999.11 \\
\hline $0818-128$ & 15.0 & 082057.462 & -125859.32 & +0.20 & $=-0.16$ & 1999.11 \\
\hline $0827+243$ & 17.3 & 083052.029 & +241100.80 & -0.78 & $=+0.98$ & 1999.14 \\
\hline $0851+202$ & 15.4 & 085448.874 & +200630.24 & -0.01 & $=-0.40$ & 1996.06 \\
\hline $1652+398$ & 13.9 & 165352.210 & +394537.33 & -0.08 & $=+0.72$ & 1999.37 \\
\hline $1727+502$ & 16.0 & 172818.635 & +501310.71 & +0.11 & $=+0.24$ & 1999.37 \\
\hline $2145+067$ & 16.5 & 214805.487 & +06 5739.37 & +0.42 & $=+0.77$ & 1996.64 \\
\hline $2200+420$ & 14.7 & 220243.292 & +421640.03 & +0.01 & $=+0.05$ & 1998.18 \\
\hline
\end{tabular}

Acknowledgments. We are indebted to the Department of International Scientific and Technical Cooperation, Ministry of Ukraine for Science and Technology and the Chinese Natural Science Foundation for their financial support this research work.

\section{References}

ESA, 1997, The Hipparcos and Tycho Catalogue, ES SP-1200.

IERS Technique Note No. 23, 1996.

Kovalchuk, A., Pinigin, G., Shulga, A., 1997, in Journées 1997 Systèmes de Référence Spatio-Temporels, Sept. 22-24, Prague, 14-17.

Ma, C., Arias, E. F., Eubanks, T. M., Fey, A. L., Gontier, A.-M., Jacobs, C. S., Sovers, O. J., Archinal, B. A., \& Charlot, P. 1998, Astron. J., 116, 516.

Mignard, F., 1998, Working Group on the International Celestial Reference System, Circular 1.

Murray, C.A., 1983, Vectorial Astrometry, Adam Hilger Ltd, Bristol, 229.

Tang, Z.H., Wang, S.H., Jin, W.J., 1998, Kinematic and Physics of Celestial Bodies, 14, 451.

Wang S.H., Tang Z.H., 1998, Kinematic and Physics of Celestial Bodies, 14, 145. 